\title{
PERISTALTIC FLOW OF REACTIVE VISCOUS FLUID WITH TEMPERATURE DEPENDENT VISCOSITY
}

\author{
S. Asghar ${ }^{1,2}$, Q. Hussain ${ }^{1}$ and T. Hayat ${ }^{2,3}$ \\ ${ }^{1}$ Department of Mathematics, COMSATS Institute of Information Technology, \\ Islamabad 44000, Pakistan \\ 2 Department of Mathematics, Faculty of Science, King Abdulaziz University, \\ P.O.Box 80203, Jeddah 21589, Saudi Arabia \\ ${ }^{3}$ Department of Mathematics, Quaid-I-Azam University 45320, Islamabad 44000, \\ Pakistan \\ sasghar@comsats.edu.pk, q.hussain@ymail.com,pensy_t@yahoo.com
}

\begin{abstract}
This article presents the analysis of peristaltic transport of reactive viscous fluid in a two-dimensional channel. We assumed that the exothermic reaction takes place within the channel under Arrhenius kinetics. The viscosity of fluid is taken temperature dependent. The nonlinear equations governing the flow are derived under the long wavelength and low Reynolds number approximations. The power series solutions are constructed in terms of small powers of the reaction parameter. The graphical results are presented and the effects of embedded parameters on the quantities of interest are examined.
\end{abstract}

Key Words- Peristaltic transport, reactive viscous fluid, variable viscosity.

\section{INTRODUCTION}

The transport of fluids from one place to any other place due to the contraction and relaxation of the channel walls is termed as peristalsis. These contractions and relaxations travel in the form of waves along the channel walls and propel the fluid. It is observed that the peristaltic flows are very common in many physiological processes such as urine transport from kidneys to bladder through ureter, movement of ovum in the female fallopian tube, circulation of blood in the small blood vessels, swallowing of food through esophagus, chyme motion in the gastrointestinal tract, movement of spermatozoa in the human reproductive tract etc. In industrial mechanics fluids like aggressive chemical, slurries, corrosive and noxious fluids in nuclear industry are transported by the peristaltic pumping. Moreover, the study of peristalsis has a key role in designing the roller pumps, finger pumps, hose pumps, dialysis machines, heart lung machines and blood pump machines. In the light of physiological and industrial applications, the mechanism of peristalsis has received special attention of the recent researchers. In fact Latham [1] was the first who investigated the mechanism of peristalsis experimentally. Soon after, Burns et al. [2] and Shapiro et al. [3] have focused their attention to the study of peristaltic flows. Burns et al. [2] discussed the peristaltic motion by considering the sinusoidal type wall deformations while Shapiro et al. [3] analyzed the peristaltic pumping in the presence of long wavelength and low Reynolds number assumptions.

Since all living beings exchange heat with the environment in almost all 
conditions and small variations in temperature can lead to an irreversible damage of the platelets and white cells of the blood. Heat transfer analysis in peristaltic flow pattern is important in oxygenation, hemodialysis and in better designing of the bio-instruments. Hence at present some studies have been reported for the peristaltic flows in the presence of heat transfer. Radhakrishnamacharya and Srinivasulu [4] analyzed the influence of wall properties and heat transfer on peristaltic pumping. Srinivas and Muthuraj [5] discussed the effects of chemical reaction by considering the peristaltic flow of electrically conducting fluid in vertical asymmetric channel. Mekheimer [6] documented the influence of heat transfer for peristaltic transport of magnetohydrodynamic (MHD) Newtonian fluid in a vertical annulus. Ali et al. [7] studied the heat transfer analysis of peristaltic flow of viscous fluid in a curved channel. Vajravelu et al. [8] discussed the effects of heat transfer on peristaltic transport of a Jeffrey fluid in a vertical porous stratum. Tripathi [9] presented a mathematical model for swallowing of food bolus through the oesophagus when heat effects are present. Further, the readers may be mentioned to the interesting studies on the interaction of heat transfer with peristalsis [10-14].

All the studies mentioned above deal with the constant viscosity fluid. The assumption of constant viscosity fluid fails when peristaltic motion in small blood vessels, lymphatic vessels and intestine is considered. The change in the fluid viscosity greatly affects the fluid properties. The physiological fluids such as blood, various lubricants like polymer solutions and mineral oils with polymer additives can be grouped as temperature dependent variable viscosity fluids. The heat transfer analysis of such kind of fluids has much applications in food processing instruments, biosciences, viscometry and lubrications. Few recent studies regarding the effects of variable viscosity on peristalsis can be found in the refs. [15-20]. Note that in all these attempts, the non-reactive fluids are considered.

Heat transfer and thermal stability of the reactive viscous fluid play a key role in order to ensure the safety of life and property during handling and processing of reactive fluids such as coal slurries, polymer solutions, drilling muds, hydrocarbon oils, grease etc. Hence the aim of present study is to develop a mathematical model addressing the peristaltic flow of reactive variable viscosity fluid in a symmetric channel. To our knowledge such analysis has not been reported so far. This study is organized as follows. The problem is formulated in section two. The solutions of the governing nonlinear analysis are obtained in section three. In section four, the graphical results are presented and discussed with respect to the involved parameters. Section five is devoted to the concluding remarks.

\section{PROBLEMS DEVELOPMENT}

We consider the two-dimensional flow of an incompressible reactive variable viscosity fluid in a symmetric channel of uniform thickness $2 d$. The channel walls have temperature $T_{w}$. The viscosity of the fluid is assumed temperature dependent. The flow is driven by propagation of the sinusoidal wave trains along the channel walls with constant speed $c$. Flow configuration in the present consideration is shown in Fig. 1. The wall deformation in rectangular coordinate system $(\tilde{X}, \tilde{Y}, \tilde{t})$ is defined by 


$$
\tilde{H}(\tilde{X}, t)=d+b \cos \left[\frac{2 \pi}{\lambda}(\tilde{X}-c \tilde{t})\right],
$$

in which $d, b, \lambda, \tilde{X}$ and $\tilde{t}$ represent the channel half width, the wave amplitude, the wavelength, the direction of the wave propagation and the time.

The unsteady flow can be treated as steady by switching from fixed frame $(\tilde{X}, \tilde{Y})$ to wave frame $(\tilde{x}, \tilde{y})$ which travels in $\tilde{X}$ - direction with the same speed of wave. For this purpose it is convenient to employ the following transformations:

$$
(\tilde{x}, \tilde{y})=(\tilde{X}-c \tilde{t}, \tilde{Y}),(\tilde{u}, \tilde{v})=(\tilde{U}-c, \tilde{V}), \tilde{p}(\tilde{x})=\tilde{P}(\tilde{X}, \tilde{t}),
$$

where $\tilde{U}, \tilde{V}$ are the velocity components in the fixed frame and $\tilde{u}, \tilde{v}$ are the velocity components in wave frame. Further $\tilde{p}$ and $\tilde{P}$ are the pressures in the fixed and wave frames respectively.

In wave frame, the continuity, momentum and energy equations in the absence of reactant fluid consumption are

$$
\begin{gathered}
\frac{\partial \tilde{u}}{\partial \tilde{x}}+\frac{\partial \tilde{v}}{\partial \tilde{y}}=0 \\
\rho\left(\tilde{u} \frac{\partial \tilde{u}}{\partial \tilde{x}}+\tilde{v} \frac{\partial \tilde{u}}{\partial \tilde{y}}\right)=-\frac{\partial \tilde{p}}{\partial \tilde{x}}+2 \frac{\partial}{\partial \tilde{x}}\left(\tilde{\mu} \frac{\partial \tilde{u}}{\partial \tilde{x}}\right)+\frac{\partial}{\partial \tilde{y}}\left[\tilde{\mu}\left(\frac{\partial \tilde{v}}{\partial \tilde{x}}+\frac{\partial \tilde{u}}{\partial \tilde{y}}\right)\right], \\
\rho\left(\tilde{u} \frac{\partial \tilde{v}}{\partial \tilde{x}}+\tilde{v} \frac{\partial \tilde{v}}{\partial \tilde{y}}\right)=-\frac{\partial \tilde{p}}{\partial \tilde{y}}+2 \frac{\partial}{\partial \tilde{y}}\left(\tilde{\mu} \frac{\partial \tilde{v}}{\partial \tilde{y}}\right)+\frac{\partial}{\partial \tilde{x}}\left[\tilde{\mu}\left(\frac{\partial \tilde{v}}{\partial \tilde{x}}+\frac{\partial \tilde{u}}{\partial \tilde{y}}\right)\right], \\
\rho\left(\tilde{u} \frac{\partial \tilde{T}}{\partial \tilde{x}}+\tilde{v} \frac{\partial \tilde{T}}{\partial \tilde{y}}\right)=\kappa\left(\frac{\partial^{2} \tilde{T}}{\partial \tilde{x}^{2}}+\frac{\partial^{2} \tilde{T}}{\partial \tilde{y}^{2}}\right)+S C_{0} A e^{-\frac{E}{R \tilde{T}}}
\end{gathered}
$$

In above equations $\rho, \tilde{T}, c_{p}, \kappa, S, C_{0}, A, E$ and $R$ stand for the density of the fluid, the absolute temperature, the specific heat, the thermal conductivity of the fluid, the heat of reaction, the initial concentration of the reactant species, the rate constant, the activation energy and the universal gas constant.

The temperature dependent viscosity $\tilde{\mu}$ following the Reynolds model can be expressed as

$$
\tilde{\mu}=\mu_{0} e^{-\alpha\left(\tilde{T}-T_{w}\right)},
$$

wherein $\mu_{0}$ is the constant dynamic viscosity of the fluid at $\tilde{T}=T_{w}$. 


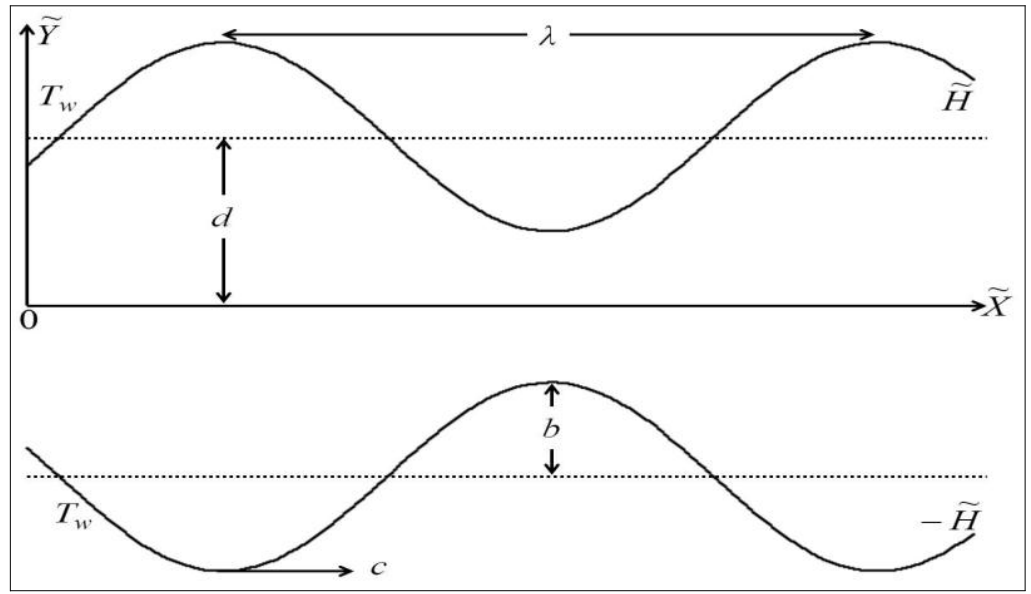

Fig. 1. Geometry of the physical model

Introducing

$$
\begin{aligned}
& x=\frac{\tilde{x}}{\lambda}, y=\frac{\tilde{y}}{d}, u=\frac{\tilde{u}}{c}, v=\frac{\tilde{v}}{c}, t=\frac{c \tilde{t}}{\lambda}, T=\frac{E\left(\tilde{T}-T_{w}\right)}{R T_{w}^{2}}, \\
& p=\frac{d^{2} \tilde{p}}{c \lambda \mu_{0}}, h=\frac{\tilde{H}}{d}, \phi=\frac{b}{d}, \mu=\frac{\tilde{\mu}}{\mu_{0}}, \delta=\frac{d}{\lambda}, \beta=\frac{\alpha R T_{w}^{2}}{E}, \\
& \operatorname{Re}=\frac{\rho c d}{\mu}, \operatorname{Pr}=\frac{\mu c_{p}}{\kappa}, \varepsilon=\frac{R T_{w}}{E}, \Gamma=\frac{S C_{0} A E d^{2} e^{-\frac{E}{R T_{w}}}}{\kappa R T_{w}^{2}},
\end{aligned}
$$

and then using the non-dimensional variables and parameters into Eqs. (3-7) we have

$$
\begin{gathered}
\delta \frac{\partial u}{\partial x}+\frac{\partial v}{\partial y}=0 \\
\operatorname{Re}\left[\delta u \frac{\partial u}{\partial x}+v \frac{\partial u}{\partial y}\right]=-\frac{\partial p}{\partial x}+2 \delta^{2} \frac{\partial}{\partial x}\left[\mu \frac{\partial u}{\partial x}\right]+\frac{\partial}{\partial y}\left[\mu\left(\delta \frac{\partial v}{\partial x}+\frac{\partial u}{\partial y}\right)\right], \\
\operatorname{Re} \delta\left[\delta u \frac{\partial v}{\partial x}+v \frac{\partial v}{\partial y}\right]=-\frac{\partial p}{\partial y}+2 \delta \frac{\partial}{\partial y}\left[\mu \frac{\partial v}{\partial y}\right]+\delta^{2} \frac{\partial}{\partial x}\left[\mu\left(\delta \frac{\partial v}{\partial x}+\frac{\partial u}{\partial y}\right)\right], \\
\operatorname{PrRe}\left[\delta u \frac{\partial T}{\partial x}+v \frac{\partial T}{\partial y}\right]=\left[\delta^{2} \frac{\partial^{2} T}{\partial x^{2}}+\frac{\partial^{2} T}{\partial y^{2}}\right]+\Gamma\left[e^{\frac{T}{1+\varepsilon T}}\right]
\end{gathered}
$$

where $\mu=e^{-\beta T}$ is the viscosity of the fluid, $\delta$ is the wave number, Re is the Reynolds number, $\operatorname{Pr}$ is the Prandtl number, $\beta$ is the viscosity parameter, $\Gamma$ is the reaction parameter (Frank Kamenetskii parameter) and $\varepsilon$ is the activation energy parameter.

Introducing the stream function by $u=\partial \psi / \partial y, \quad v=-\delta(\partial \psi / \partial x)$ and adopting the long wavelength procedure in Eqs. (9-12), we arrive at

$$
\begin{aligned}
& \frac{\partial^{2}}{\partial y^{2}}\left[\mu \frac{\partial^{2} \psi}{\partial y^{2}}\right]=0, \\
& \frac{d p}{d x}=\frac{\partial}{\partial y}\left[\mu \frac{\partial^{2} \psi}{\partial y^{2}}\right],
\end{aligned}
$$




$$
\frac{\partial^{2} T}{\partial y^{2}}+\Gamma\left[e^{\frac{T}{1+\varepsilon T}}\right]=0
$$

Note that the incompressibility condition is clearly satisfied and $\partial p / \partial y=0$ employing that $p \neq p(y)$.

The dimensionless form of the boundary conditions are given by

$$
\begin{gathered}
\psi=0, \frac{\partial^{2} \psi}{\partial^{2} y}=0, T=0, \text { at } y=0, \\
\psi=F, \frac{\partial \psi}{\partial y}=-1, T=0, \text { at } y=h=1+a \cos [2 \pi x],
\end{gathered}
$$

where

$$
F=\int_{0}^{h} \frac{\partial \psi}{\partial y} d y
$$

The respective dimensionless mean flow rates $Q$ and $F$ in the laboratory and wave frames can be related as follows

$$
Q=F+1 .
$$

\section{SOLUTION PROCEDURE}

Clearly the Eqs. (13-15) governing the fluid motion and heat transfer comprise nonlinear situation. Closed form analytic solutions of the involved problems are not possible. Hence the series expressions are constructed for small reaction parameter $\Gamma$. Thus we expand the quantities as follows:

$$
\begin{aligned}
& \psi=\psi_{0}+\Gamma \psi_{1}+\Gamma^{2} \psi_{2}+\Gamma^{3} \psi_{3}+O\left(\Gamma^{4}\right), \\
& T=T_{0}+\Gamma T_{1}+\Gamma^{2} T_{2}+\Gamma^{3} T_{3}+O\left(\Gamma^{4}\right), \\
& p=p_{0}+\Gamma p_{1}+\Gamma^{2} p_{2}+\Gamma^{3} p_{3}+O\left(\Gamma^{4}\right), \\
& F=F_{0}+\Gamma F_{1}+\Gamma^{2} F_{2}+\Gamma^{3} F_{3}+O\left(\Gamma^{4}\right) .
\end{aligned}
$$

Using Eqs. (20-23) into Eqs.(13-17) and collecting the coefficients of like powers of $\Gamma$, we have the following systems:

\section{Zeroth order system}

$$
\begin{gathered}
\frac{\partial^{2}}{\partial y^{2}}\left[e^{-\beta T_{0}} \frac{\partial^{2} \psi_{0}}{\partial y^{2}}\right]=0, \\
\frac{d p_{0}}{d x}=\frac{\partial}{\partial y}\left[e^{-\beta T_{0}} \frac{\partial^{2} \psi_{0}}{\partial y^{2}}\right], \\
\frac{\partial^{2} T_{0}}{\partial y^{2}}=0, \\
\psi_{0}=0, \frac{\partial^{2} \psi_{0}}{\partial y^{2}}=0, T_{0}=0 \text { at } y=0, \psi_{0}=F_{0}, \frac{\partial \psi_{0}}{\partial y}=-1, T_{0}=0 \text { at } y=h .
\end{gathered}
$$

First order system

$$
\frac{\partial^{2}}{\partial y^{2}}\left[e^{-\beta T_{0}}\left(\frac{\partial^{2} \psi_{1}}{\partial y^{2}}-\beta T_{1} \frac{\partial^{2} \psi_{0}}{\partial y^{2}}\right)\right]=0,
$$




$$
\begin{gathered}
\frac{d p_{0}}{d x}=\frac{\partial}{\partial y}\left[e^{-\beta T_{0}}\left(\frac{\partial^{2} \psi_{1}}{\partial y^{2}}-\beta T_{1} \frac{\partial^{2} \psi_{0}}{\partial y^{2}}\right)\right], \\
\frac{\partial^{2} T_{1}}{\partial y^{2}}+e^{\frac{T_{0}}{1+\varepsilon_{0}}}=0, \\
\psi_{1}=0, \frac{\partial^{2} \psi_{1}}{\partial y^{2}}=0, T_{1}=0 \text { at } y=0, \psi_{1}=F_{1}, \frac{\partial \psi_{1}}{\partial y}=0, T_{1}=0 \text { at } y=h .
\end{gathered}
$$

\section{Second order system}

$$
\begin{aligned}
& \frac{\partial^{2}}{\partial y^{2}}\left[e^{-\beta T_{0}}\left(\frac{\partial^{2} \psi_{2}}{\partial y^{2}}-\beta T_{1} \frac{\partial^{2} \psi_{1}}{\partial y^{2}}+\frac{1}{2} \beta\left(\beta T_{1}-2 T_{2}\right) \frac{\partial^{2} \psi_{0}}{\partial y^{2}}\right)\right]=0, \\
& \frac{d p_{2}}{d x}=\frac{\partial}{\partial y}\left[e^{-\beta T_{0}}\left(\frac{\partial^{2} \psi_{2}}{\partial y^{2}}-\beta T_{1} \frac{\partial^{2} \psi_{1}}{\partial y^{2}}+\frac{1}{2} \beta\left(\beta T_{1}-2 T_{2}\right) \frac{\partial^{2} \psi_{0}}{\partial y^{2}}\right)\right], \\
& \frac{\partial^{2} T_{1}}{\partial y^{2}}+\frac{e^{\frac{T_{0}}{1+\varepsilon T_{0}}} T_{1}}{\left(1+\varepsilon T_{0}\right)^{2}}=0 \\
& \psi_{2}=0, \frac{\partial^{2} \psi_{2}}{\partial y^{2}}=0, T_{2}=0 \text { at } y=0, \psi_{2}=F_{2}, \frac{\partial \psi_{2}}{\partial y}=0, T_{2}=0 \text { at } y=h .
\end{aligned}
$$

\section{Third order system}

$$
\begin{aligned}
& \frac{\partial^{2}}{\partial y^{2}}\left[e^{-\beta T_{0}}\left(\begin{array}{c}
\frac{\partial^{2} \psi_{3}}{\partial y^{2}}-\beta T_{1} \frac{\partial^{2} \psi_{2}}{\partial y^{2}}+\frac{1}{2} \beta\left(\beta T_{1}-2 T_{2}\right) \frac{\partial^{2} \psi_{1}}{\partial y^{2}} \\
+\frac{1}{6} \beta\left(\beta^{2} T_{1}^{3}-6 \beta T_{1} T_{2}+6 T_{3}\right) \frac{\partial^{2} \psi_{0}}{\partial y^{2}}
\end{array}\right)\right]=0, \\
& \frac{d p_{3}}{d x}=\frac{\partial}{\partial y}\left[e^{-\beta T_{0}}\left(\begin{array}{c}
\frac{\partial^{2} \psi_{3}}{\partial y^{2}}-\beta T_{1} \frac{\partial^{2} \psi_{2}}{\partial y^{2}}+\frac{1}{2} \beta\left(\beta T_{1}-2 T_{2}\right) \frac{\partial^{2} \psi_{1}}{\partial y^{2}} \\
+\frac{1}{6} \beta\left(\beta^{2} T_{1}^{3}-6 \beta T_{1} T_{2}+6 T_{3}\right) \frac{\partial \partial_{0}^{2} \psi_{0}}{\partial y^{2}}
\end{array}\right)\right], \\
& \frac{\partial^{2} T_{3}}{\partial y^{2}}+e^{\frac{T_{0}}{1+\varepsilon T_{0}}}\left(\frac{T_{1}}{2\left(1+\varepsilon T_{0}\right)^{4}}+\frac{T_{2}-\varepsilon\left(T_{1}^{2}-T_{0} T_{2}\right)}{\left(1+\varepsilon T_{0}\right)^{3}}\right)=0, \\
& \psi_{3}=0, \frac{\partial^{2} \psi_{3}}{\partial y^{2}}=0, T_{3}=0 \text { at } y=0, \psi_{3}=F_{3}, \frac{\partial \psi_{3}}{\partial y}=0, T_{3}=0 \text { at } y=h \text {. }
\end{aligned}
$$

Solving the above systems and then using $F_{0}=F-\Gamma F_{1}-\Gamma^{2} F_{2}-\Gamma^{3} F_{3}$, the expressions of the stream function, axial pressure gradient and temperature profile upto $O\left(\Gamma^{4}\right)$ may be expressed as follows:

$$
\begin{aligned}
\psi= & \frac{h^{2}(3 F+h) y-(F+h) y^{3}}{2 h^{3}}+\frac{3(F+h)\left(h^{2}-y^{2}\right)^{2} y \beta}{40 h^{3}} \\
& \times\left\{\Gamma+\frac{\left(h^{2}(265+39 \beta)-25(1+3 \beta) y^{2}\right) \Gamma^{2}}{630}-\frac{\left(3 A_{1} h^{4}+50 A_{2} h^{2} y^{2}-175 A_{3} y^{4}\right) \Gamma^{3}}{226800}\right\}, \\
\frac{d p}{d x}= & -\frac{3(F+h)}{h^{3}}\left\{1-\frac{h^{2} \beta}{5}\left(\Gamma+\frac{2(20-3 \beta) h^{2} \Gamma^{2}}{105}+\frac{(1100-3 \beta(65-2 \beta)-750 \varepsilon) h^{4} \Gamma^{3}}{4725}\right)\right\}, \\
T= & \frac{\left(h^{2}-y^{2}\right)}{2}\left\{\Gamma+\frac{\left(5 h^{2}-y^{2}\right) \Gamma^{2}}{12}+\frac{\left((47-33 \varepsilon) h^{4}-(13-12 \varepsilon) h^{2} y^{2}+(2-3 \varepsilon) y^{4}\right) \Gamma^{3}}{180}\right\},
\end{aligned}
$$

with 


$$
A_{1}=3 \beta(7 \beta-1385)+50(279 \varepsilon-398), A_{2}=152+\beta(489+57 \beta)-138 \varepsilon, A_{3}=4+15 \beta(1+\beta)-6 \varepsilon,
$$

The heat transfer coefficient at $y=h$ is

$$
Z=\left(\frac{\partial h}{\partial x}\right)\left(\frac{\partial T}{\partial y}\right)
$$

The pressure rise per wavelength $\Delta P_{\lambda}$ and frictional force $F_{\lambda}$ at the wall are given by

$$
\begin{aligned}
& \Delta P_{\lambda}=\int_{0}^{1}\left(\frac{d p}{d x}\right) d x, \\
& F_{\lambda}=\int_{0}^{1}\left(-h \frac{d p}{d x}\right) d x,
\end{aligned}
$$

where $d p / d x$ is given in Eq. (25).

\section{ANALYSIS OF RESULTS}

In this section we focused our attention to analyze the effects of involved physical parameters in the flow and heat transfer. Note that the integrals in Eqs. (28) and (29) are evaluated numerically through integration in Mathematica. 8 and results are displayed in the Figs. (2-10).

Eq. (26) is used to draw Fig. 2 which represents the effects of reaction parameter $\Gamma$ and activation parameter $\varepsilon$ on temperature profile $T$. Fig. 2(i) shows that the amplitude of temperature profile $T$ increases by increasing the reaction parameter $\Gamma$. This depicts that an increase in the reaction parameter $\Gamma$ strengthens the reaction term in the temperature equation which results an increase in the amplitude of temperature profile $T$. On the other hand, increasing values of activation energy parameter $\varepsilon$ decreases the amplitude of the temperature profile $T$ (see Fig. 2(ii)). Since $\varepsilon$ is involved in the exponential term in the temperature equation so it represents a decreasing function of the activation energy parameter $\varepsilon$. Therefore, as expected the maximum temperature is recorded in Fig. 2(ii) when $\varepsilon=0$.

Fig. 3 presents the variations of $\Gamma$ and $\varepsilon$ on heat transfer coefficient $Z$ at the wall $(y=h)$. After examining Fig. 3 we deduce that the heat transfer coefficient $Z$ has an oscillatory behavior which may be due peristalsis, that is, the heat transfer coefficient is negative for negative values of $x$ and positive for positive values of $x$. The absolute value of the heat transfer coefficient $|Z|$ increases by increasing reaction parameter $\Gamma$ while it decreases by increasing activation energy parameter $\varepsilon$. In general, $|Z|$ increases/decreases for those parameters against which fluid temperature increases/decreases .

Expression of the velocity profile $u$ is obtained by inserting the Eq. (24) into $u=\partial \psi / \partial y$ and the plots of velocity profile for various values of the $\beta, \Gamma$ and $\varepsilon$ are displayed in Figs. 4(i) to 4(iii). In Fig. 4(i) the effects of viscosity parameter $\beta$ are shown. We examined that the amplitude of velocity profile $u$ increases by increasing the viscosity parameter $\beta$. This is due to the fact that the viscosity is an inverse function of $\beta$. An increase in $\beta$ leads to a decrease in the viscosity of fluid and as a result the amplitude of the velocity $u$ increases. As seen in Fig. 2(i) that the reaction parameter $\Gamma$ rise up the fluid temperature $T$. An increased temperature decreases the fluid viscosity 
which in turn increases the amplitude of the velocity profile $u$ as shown in Fig. 4(ii) An opposite behavior of the velocity profile $u$ is shown in Fig. 4(iii) where a fall in the amplitude of velocity profile $u$ is seen for the case of increasing activation energy parameter $\varepsilon$.

The purpose of Fig. 5 is to examine the effects of $\beta, \Gamma$ and $\varepsilon$ on pressure drop $\Delta P_{\lambda}$ with $Q$. It is quite clear that there exists a critical value $Q_{0}$ (free pumping flux) against which pressure drop is zero $\left(\Delta P_{\lambda}=0\right)$. The peristaltic pumping occurs when $Q<Q_{0}$ and augmented pumping arises for $Q>Q_{0}$. The variation of $\Delta P_{\lambda}$ with $Q$ for different values of viscosity parameter $\beta$ is presented in Fig. 5(i). We observed that the free pumping flux $Q_{0}$ increases by increasing the viscosity parameter $\beta$ (Table. 1). Moreover the maximum pressure against which peristalsis works as a pump, $\Delta P_{\lambda}$ such that $Q=0$, decreases due to an increase in $\beta$. This shows that for constant viscosity fluid the peristalsis has to work against the greater pressure to push the fluid when compared with the variable viscosity fluid. The effects of reaction parameter $\Gamma$ on $\Delta P_{\lambda}$ with $Q$ can be seen through Fig. 5(ii) from where we concluded that both viscosity and reaction parameters have more or less similar impact on $\Delta P_{\lambda}$ (Table. 1). Furthermore, the peristalsis works against the greater pressure to push the fluid for non-reactive fluid when compared with the reactive fluid. Influence of activation energy parameter $\varepsilon$ is shown in Fig. 5(iii) which describes that the free pumping flux $Q_{0}$ and maximum pressure against which peristalsis works as pump decreases due to an increase in the activation energy parameter $\varepsilon$ (Table. 1). This explains that peristaltic pumping region $\left(\Delta P_{\lambda}>0, Q>0\right)$ decreases by increasing activation energy parameter $\varepsilon$.

Fig. 6 is drawn to analyze the influence of increasing $\beta, \Gamma$ and $\varepsilon$ on friction force $F_{\lambda}$ with $Q$. This Fig. illustrates that the friction force $F_{\lambda}$ shows a reverse behavior when compared with the pressure drop $\Delta P_{\lambda}$. Moreover, there also exists a critical value $Q_{h}$ against which one can define that the friction force $F_{\lambda}$ resists the flow when $Q<Q_{h}$ and it assists the flow when $Q>Q_{h}$. After examining Fig. 6 we concluded that critical value $Q_{h}$ increases when either of $\beta$ or $\Gamma$ is increased. However, critical value $Q_{h}$ decreases with increasing activation energy parameter $\varepsilon$ (Table. 1).

The variations in axial pressure gradient $d p / d x$ within one wavelength $x \in[-0.5,0.5]$ are presented in Fig. 7. This Fig. illustrates that the pressure gradient $d p / d x$ is small in the wider part of the channel $(x=0)$. This depicts that the fluid can easily flow without the imposition of large pressure gradient. Moreover, in this part of the channel $d p / d x$ negative and increases with an increase in viscosity parameter $\beta$ (Fig. 7(i)). On the other hand the pressure gradient $d p / d x$ is large in the narrow part of the channel $(x=-0.5,0.5)$ which express that a much larger pressure is required to move the fluid with same flux. In this part of the channel $d p / d x$ is positive and decreases due to an increase in $\beta$ (Fig. 7(i)). The effects of increasing reaction parameter $\Gamma$ on $d p / d x$ can be seen through Fig. 7(ii). Here we noticed that the effects of $\Gamma$ on $d p / d x$ are copy of the effects of $\beta$. We prepared Fig. 7(iii) to analyze the behavior of activation energy parameter $\varepsilon$ on $d p / d x$. This Fig. depicts that an increase in $\varepsilon$ leads to a decrease in 
$d p / d x$ in the wider part of the channel. However, no variation in $d p / d x$ with $\varepsilon$ occurs in the narrow part of the channel .

Trapping is another interesting phenomenon of peristalsis in which streamlines under certain conditions split to trap a bolus. This trapped bolus pushed ahead along with the peristaltic wall with the likewise speed of wave. The effects of $\beta, \Gamma$ and $\varepsilon$ on trapping can be seen through the Figs. (8-10). Fig. 8 displays that the volume of trapped bolus increases with an increase in the viscosity parameter $\beta$. This observation also remains true for Fig. 9 which depicts the effects of reaction parameter $\Gamma$ on trapping. Streamlines patterns in Fig. 10 indicate that the volume of the trapped bolus decreases due to an increase in the values of the activation energy parameter $\varepsilon$.

\section{CONCLUDING REMARKS}

The analysis of peristaltic transport of reactive variable viscosity fluid with heat transfer is presented. The viscosity of fluid is considered an inverse function of temperature. Novel features of the peristaltic transport and heat transfer are discussed with respect to the viscosity parameter, reaction parameter and activation energy parameter. On the basis of present study the main observations are listed below.

- The amplitude of temperature $T$ increases with increasing reaction parameter $\Gamma$ and decreases by increasing activation energy parameter $\varepsilon$.

- The absolute value of heat transfer coefficient $|Z|$ increases/decreases when temperature $T$ increases/decreases.

- An increase in either $\beta$ or in $\Gamma$ increases the amplitude of velocity $u$. However it decreases with increasing $\varepsilon$.

- For non-reactive and constant viscosity fluid the peristalsis has to work against greater pressure when compared with the reactive and variable viscosity fluid .

- The value of free pumping flux for the reactive and variable constant viscosity fluid is greater than the case of non-reactive and constant viscosity fluid.

- The peristaltic pumping region narrows down in response to the increasing activation energy parameter $\varepsilon$.

- In the wider part of the channel $d p / d x$ increases in response to the increasing $\beta$ and $\Gamma$ whereas it decreases in the narrow part of the channel. Further, the variations in $d p / d x$ with $\varepsilon$ occur only in the wider part of the channel where it decreases with increasing $\varepsilon$.

- The volume of trapped bolus increases by increasing $\beta$ and $\Gamma$ whereas it decreases by increasing $\varepsilon$. 

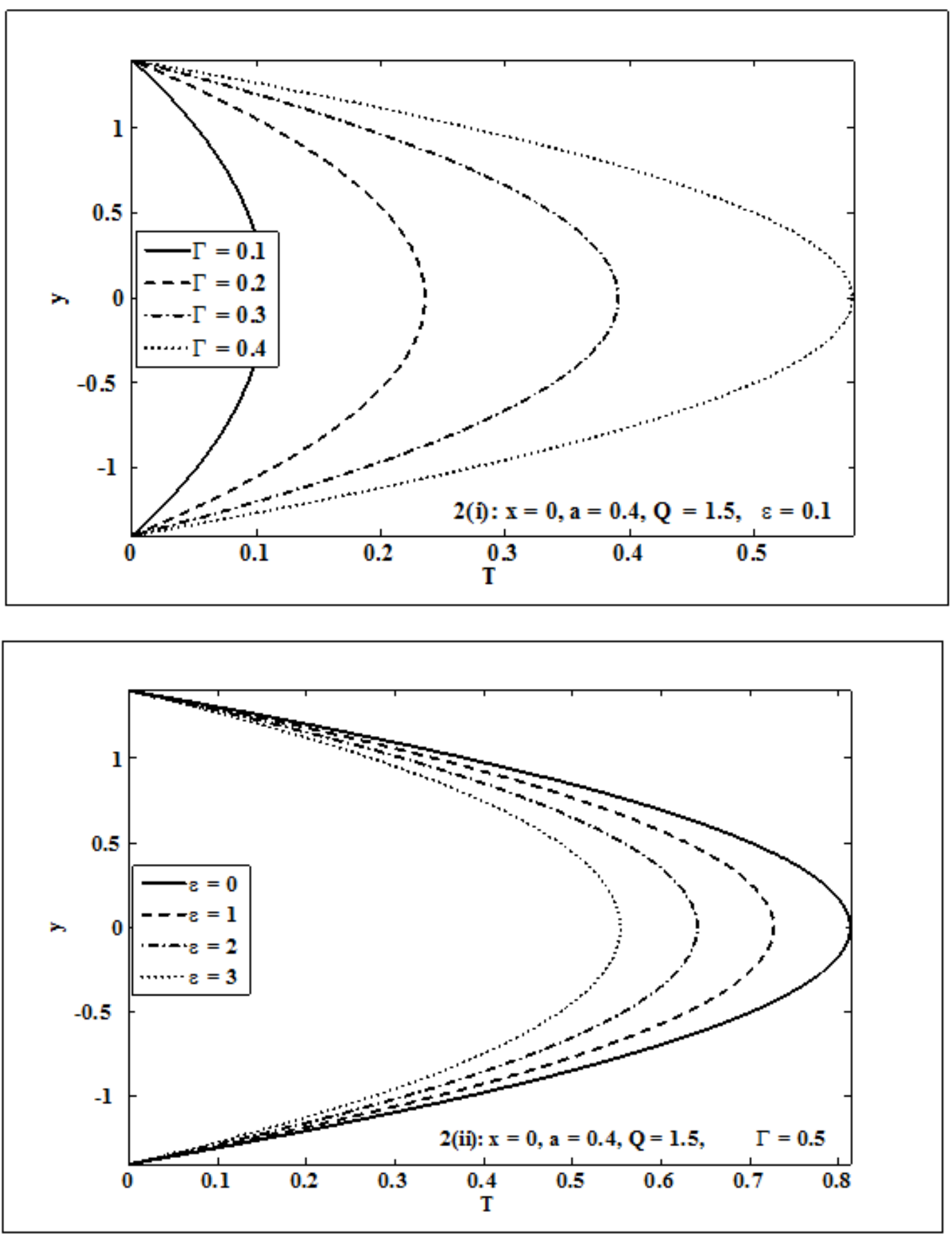

Figure 2. Plots of temperature distribution $T$ versus $y$. 

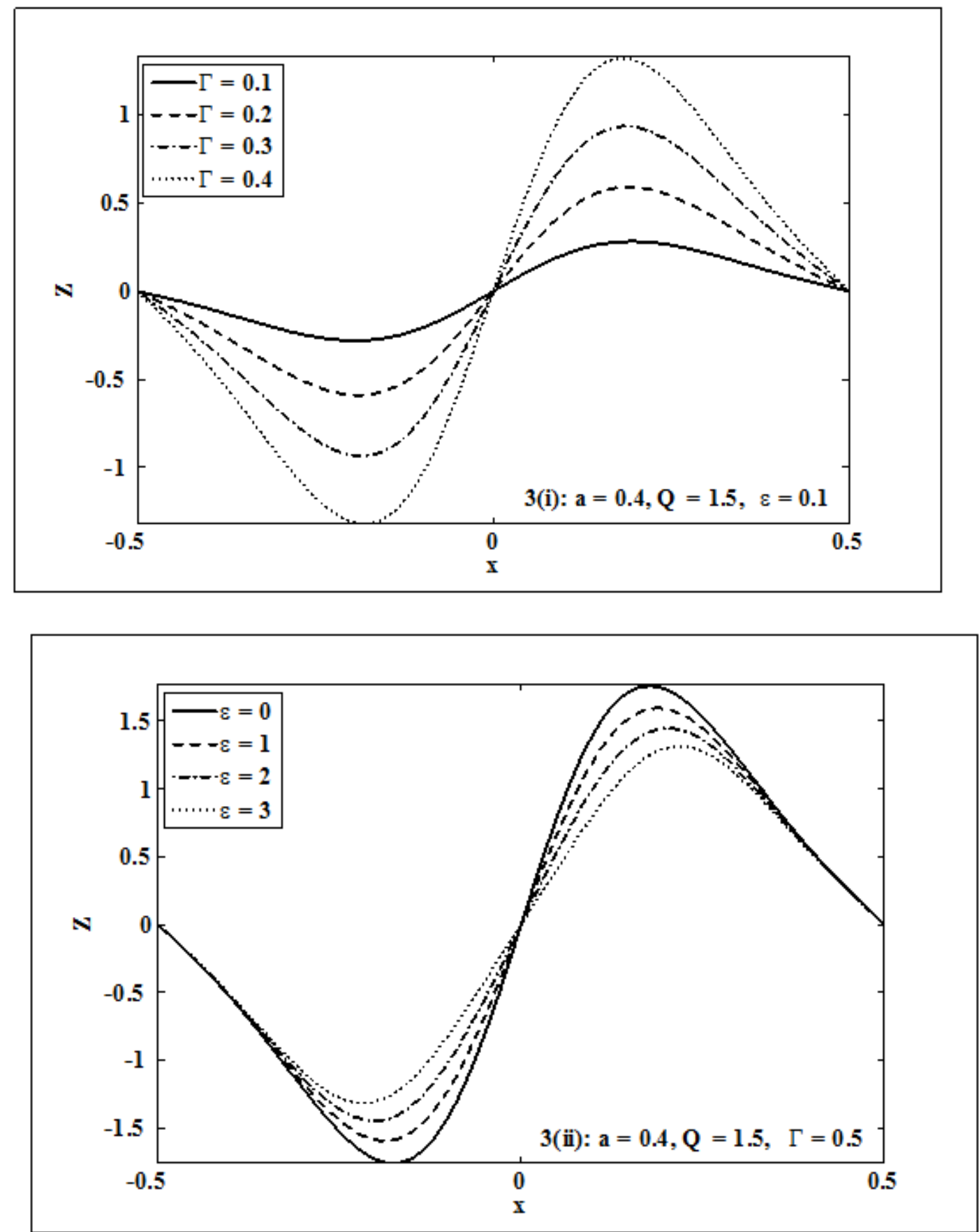

Figure 3. Plots of heat transfer coefficient at the wall $Z$ versus $x \in[-0.5,0.5]$. 

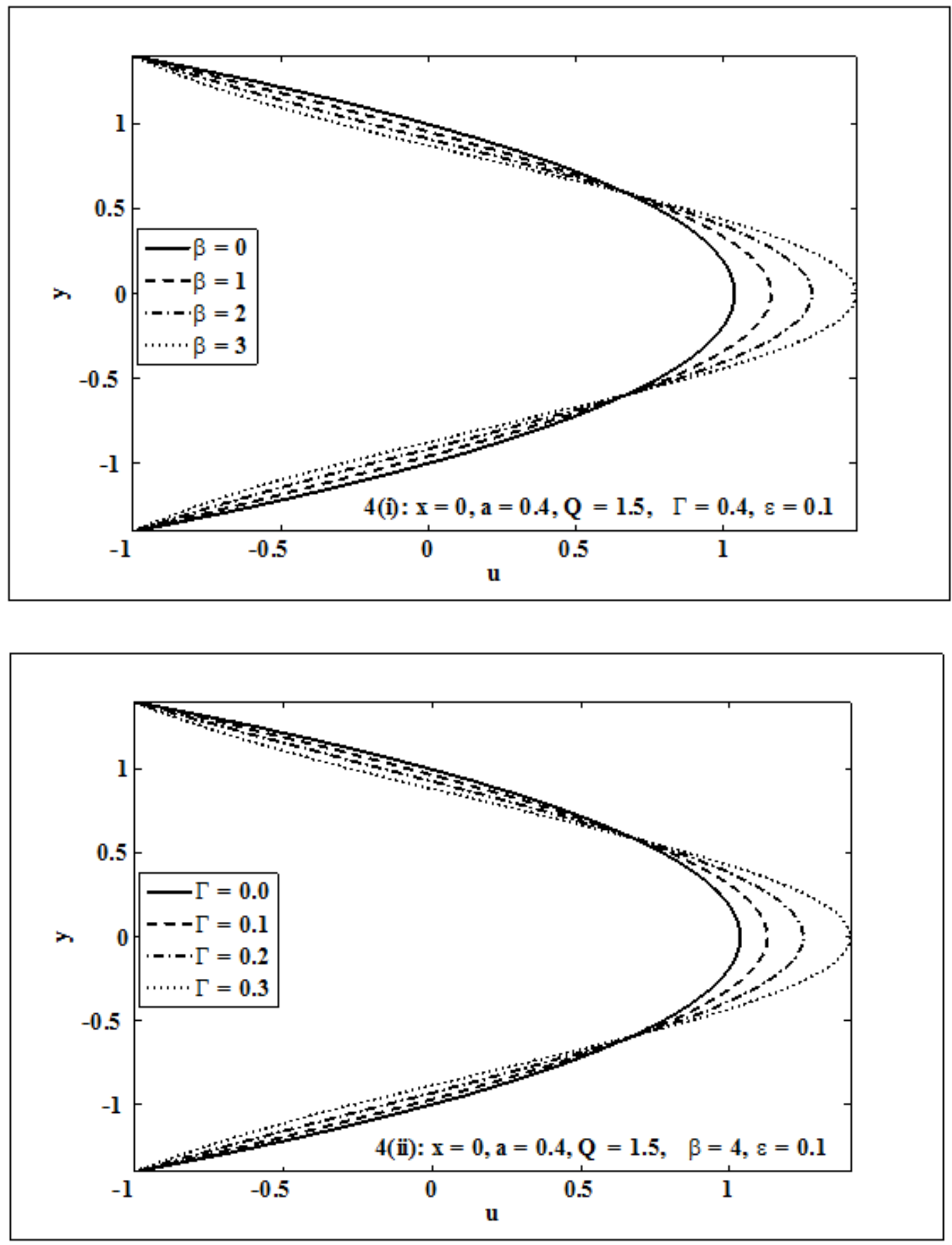


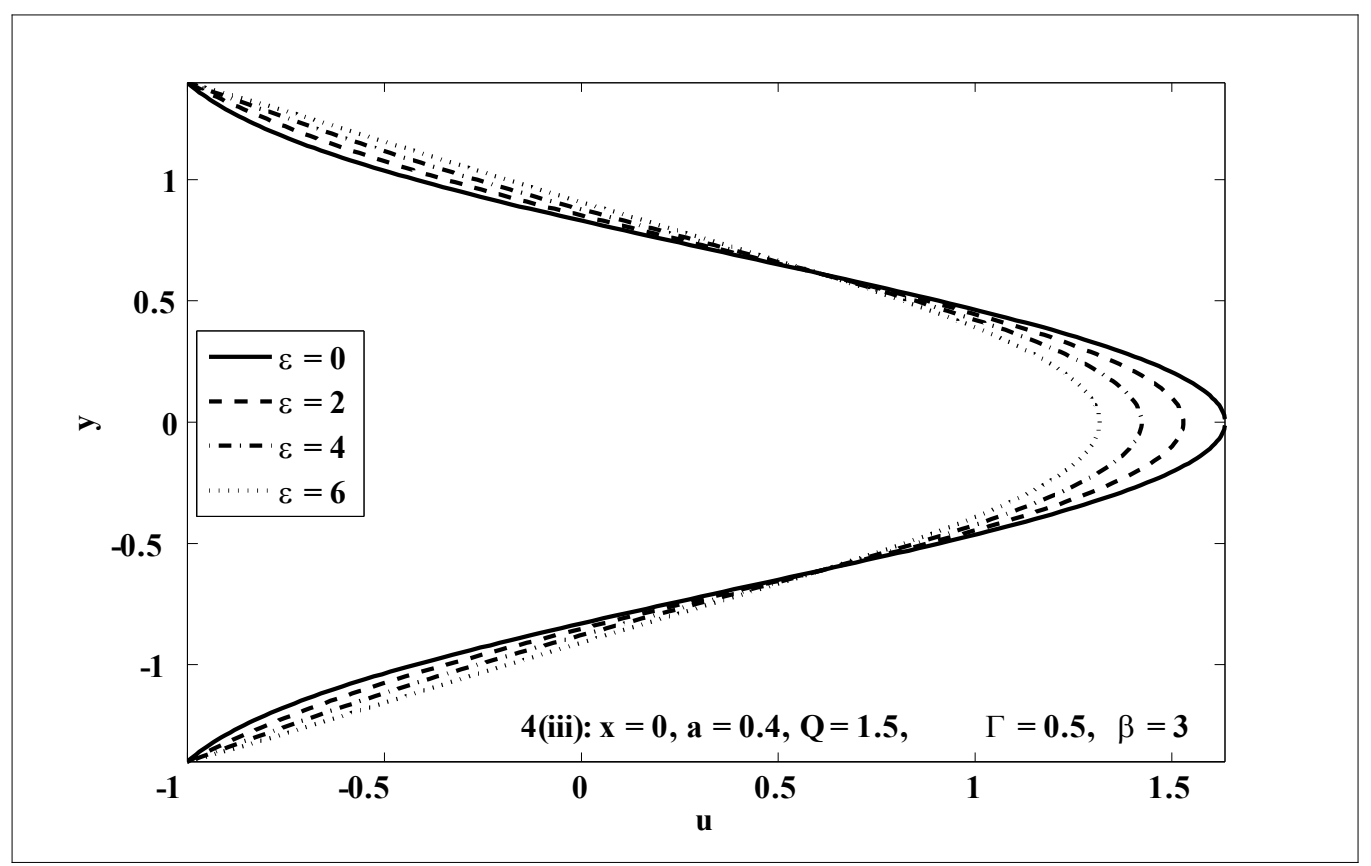

Figure 4. Plots of velocity distribution $u$ versus $y$.

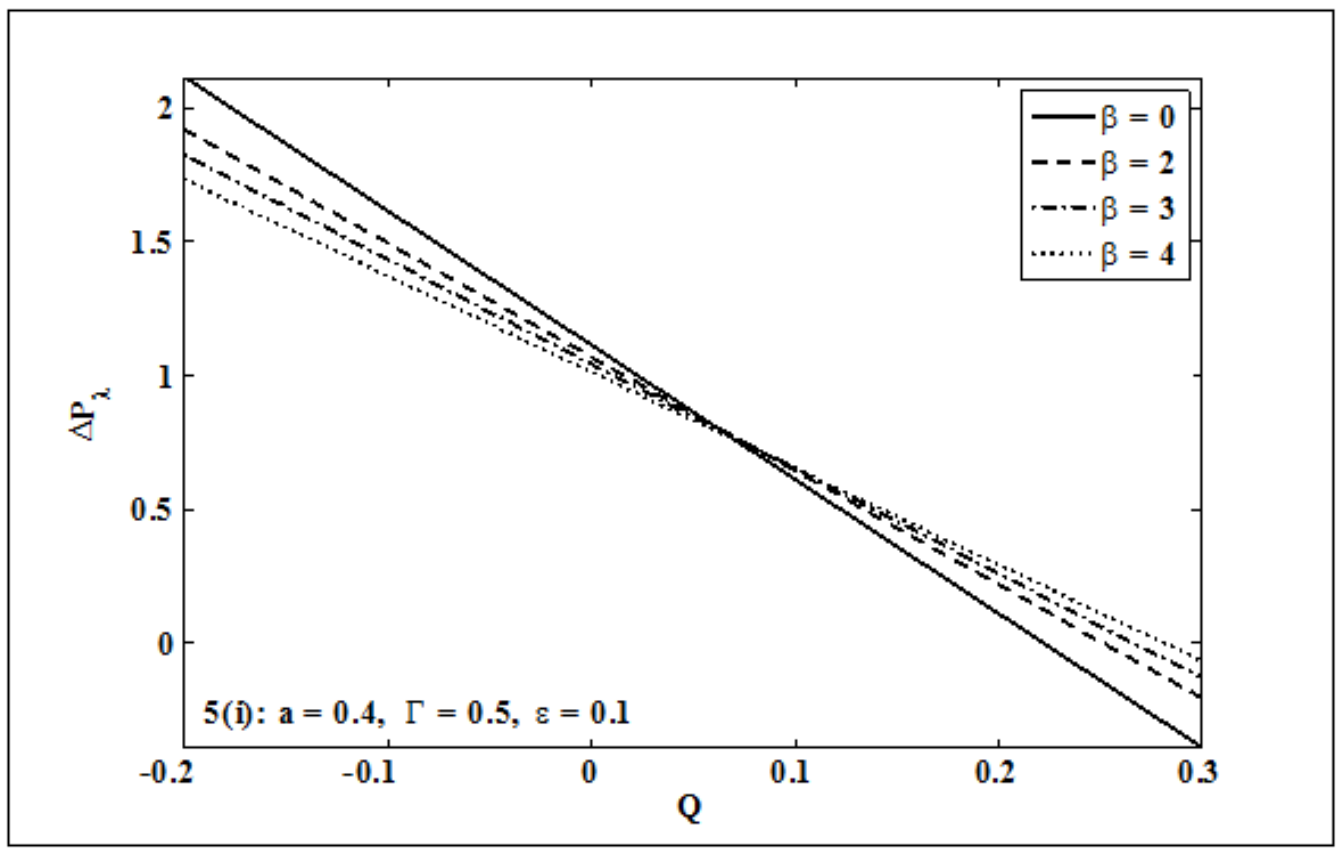



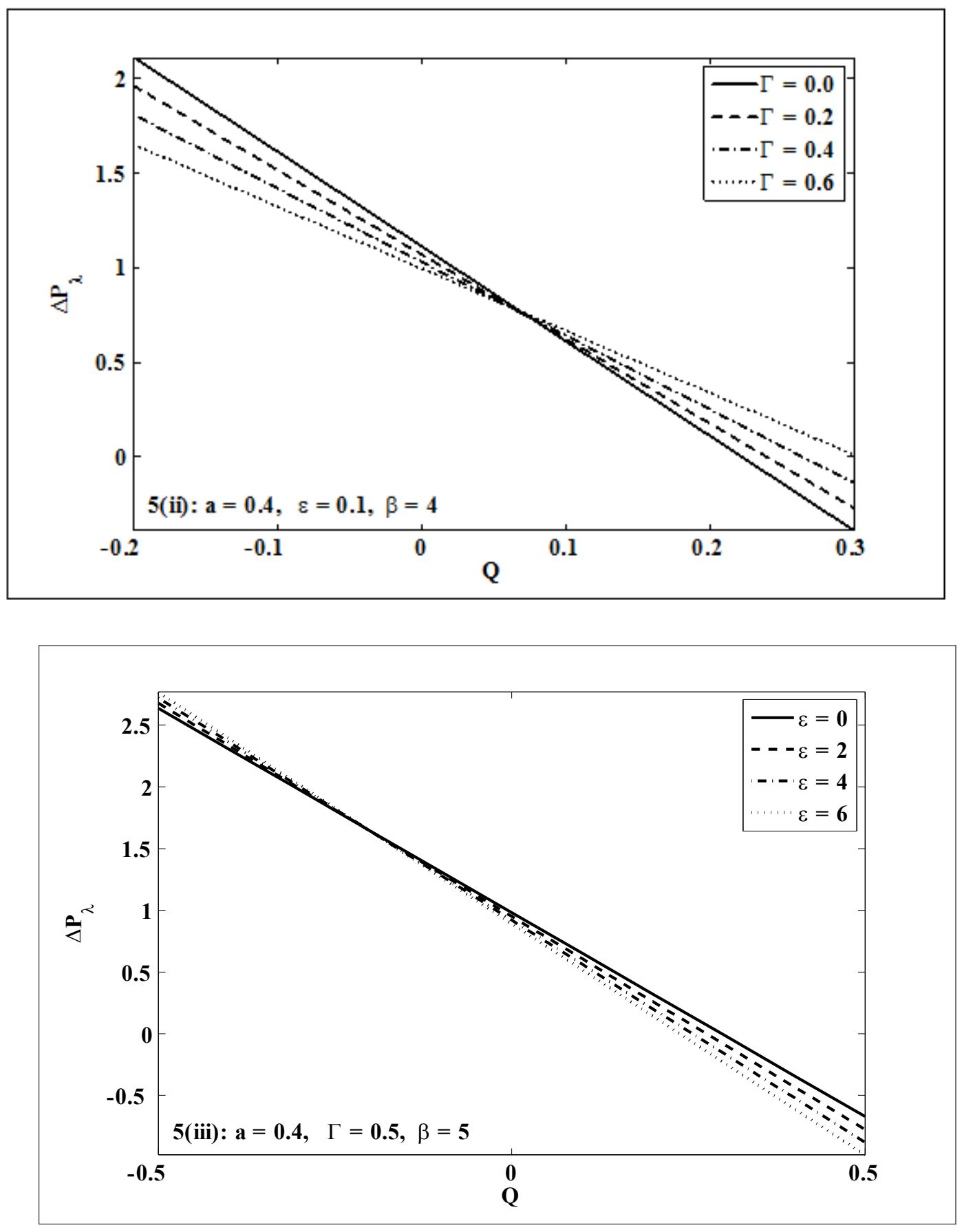

Figure 5. Plots of pressure drop per wavelength $\Delta P_{\lambda}$ versus flow rate $Q$. 

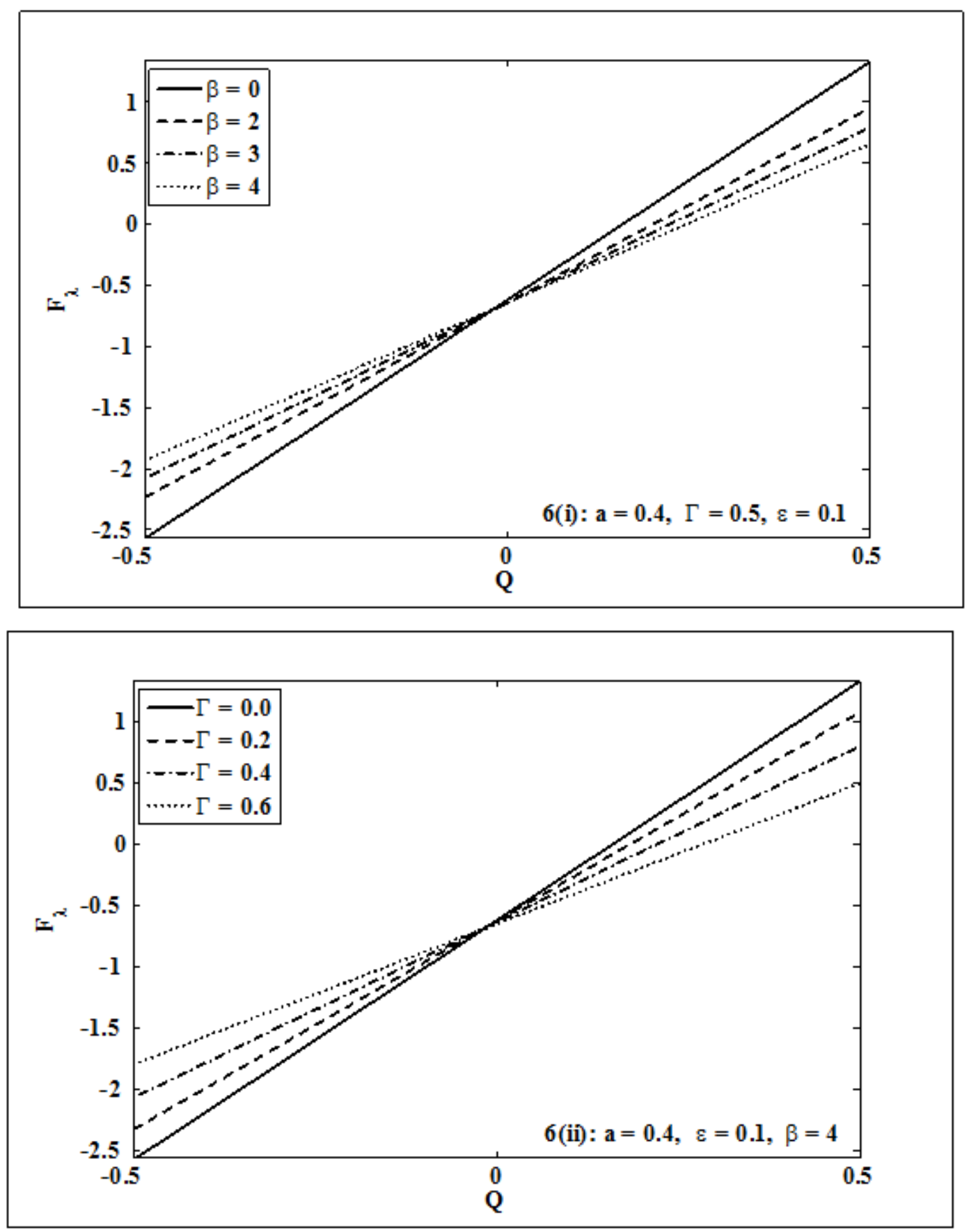


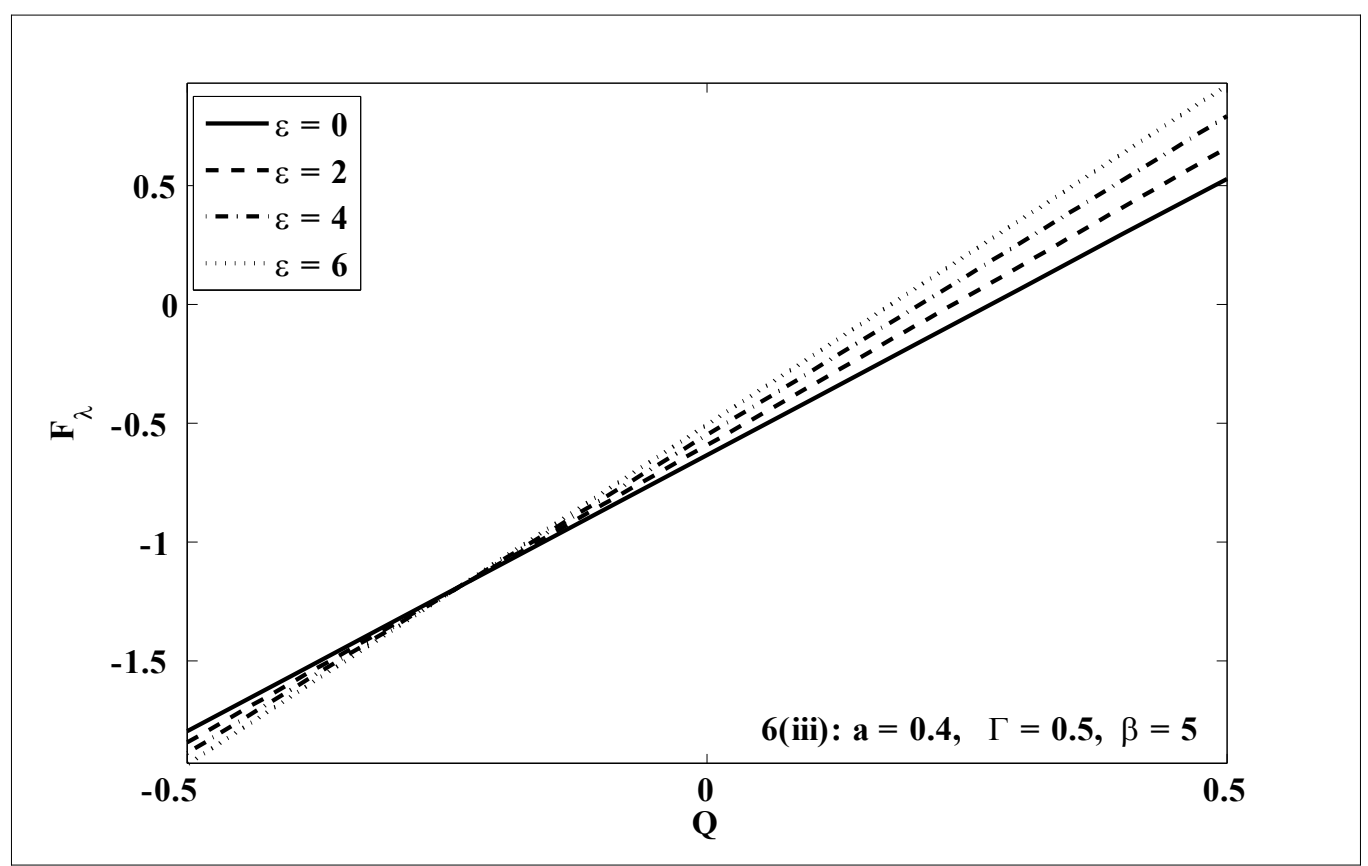

Figure 6. Plots of friction force per wavelength $F_{\lambda}$ versus flow rate $Q$.

Table 1. Critical values $Q_{0}$ against which $\Delta P_{\lambda}=0$ and $Q_{h}$ against which $F_{\lambda}=0$.

\begin{tabular}{|c|c|c|c|c|c|}
\hline Parameter $\beta$ & Critical value of $Q$ & Parameter $\Gamma$ & Critical value of $Q$ & Parameter $\varepsilon$ & Critical value of $Q$ \\
\hline \multirow{2}{*}{0} & $Q_{0}=0.2222$ & \multirow{2}{*}{0.0} & $Q_{0}=0.2222$ & \multirow{2}{*}{0} & $Q_{0}=0.2972$ \\
\hline & $Q_{h}=0.1600$ & & $Q_{h}=0.1709$ & & $Q_{h}=0.2731$ \\
\hline \multirow{2}{*}{2} & $Q_{0}=0.2517$ & \multirow{2}{*}{0.2} & $Q_{0}=0.2395$ & \multirow{2}{*}{2} & $Q_{0}=0.2758$ \\
\hline & $Q_{h}=0.2022$ & & $Q_{h}=0.1841$ & & $Q_{h}=0.2367$ \\
\hline \multirow{2}{*}{3} & $Q_{0}=0.2668$ & \multirow{2}{*}{0.4} & $Q_{0}=0.2647$ & \multirow{2}{*}{4} & $Q_{0}=0.2563$ \\
\hline & $Q_{h}=0.2250$ & & $Q_{h}=0.2006$ & & $Q_{h}=0.2051$ \\
\hline \multirow{2}{*}{4} & $Q_{0}=0.2818$ & \multirow{2}{*}{0.6} & $Q_{0}=0.3033$ & \multirow{2}{*}{6} & $Q_{0}=0.2382$ \\
\hline & $Q_{h}=0.2482$ & & $Q_{h}=0.2214$ & & $Q_{h}=0.1774$ \\
\hline
\end{tabular}



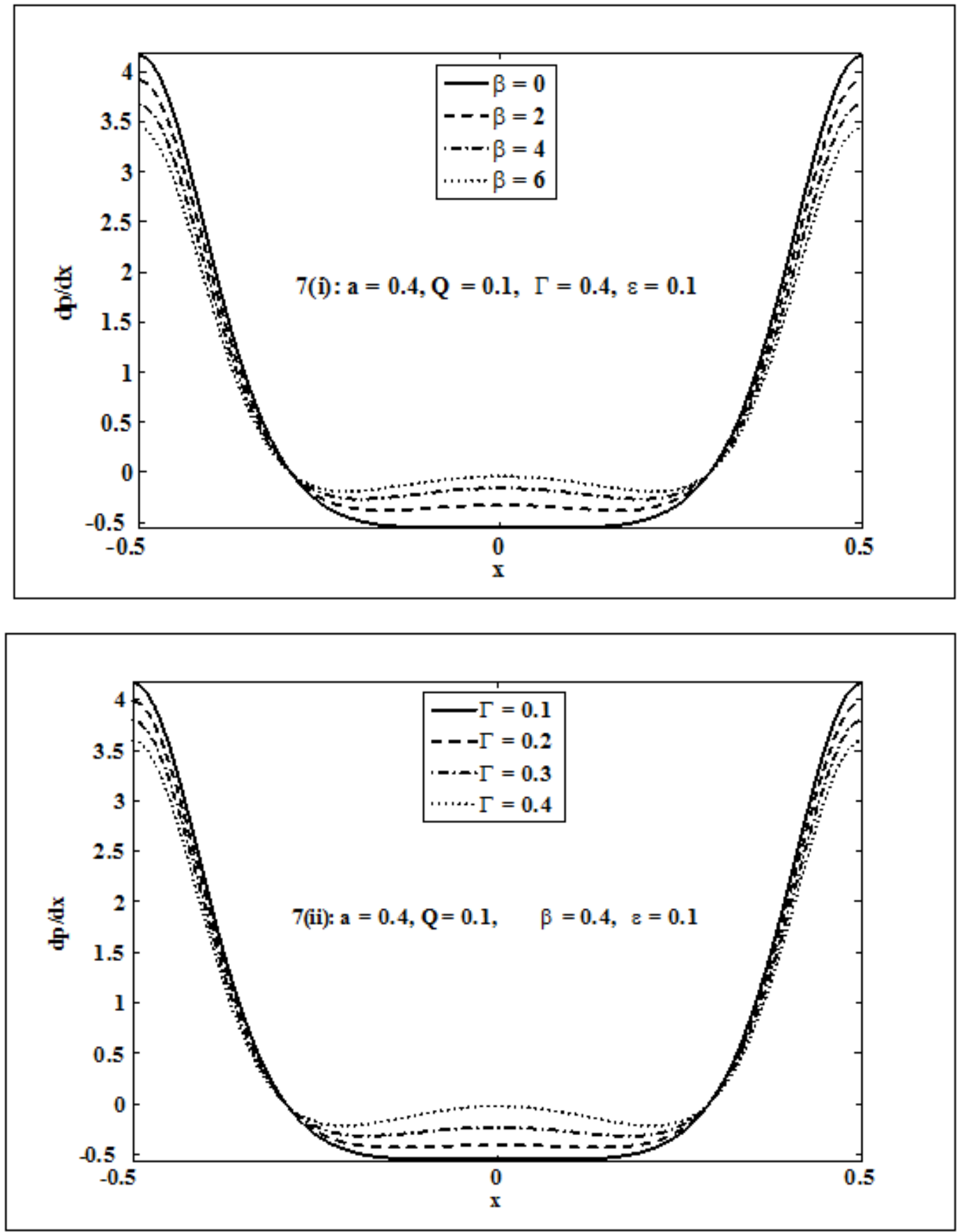


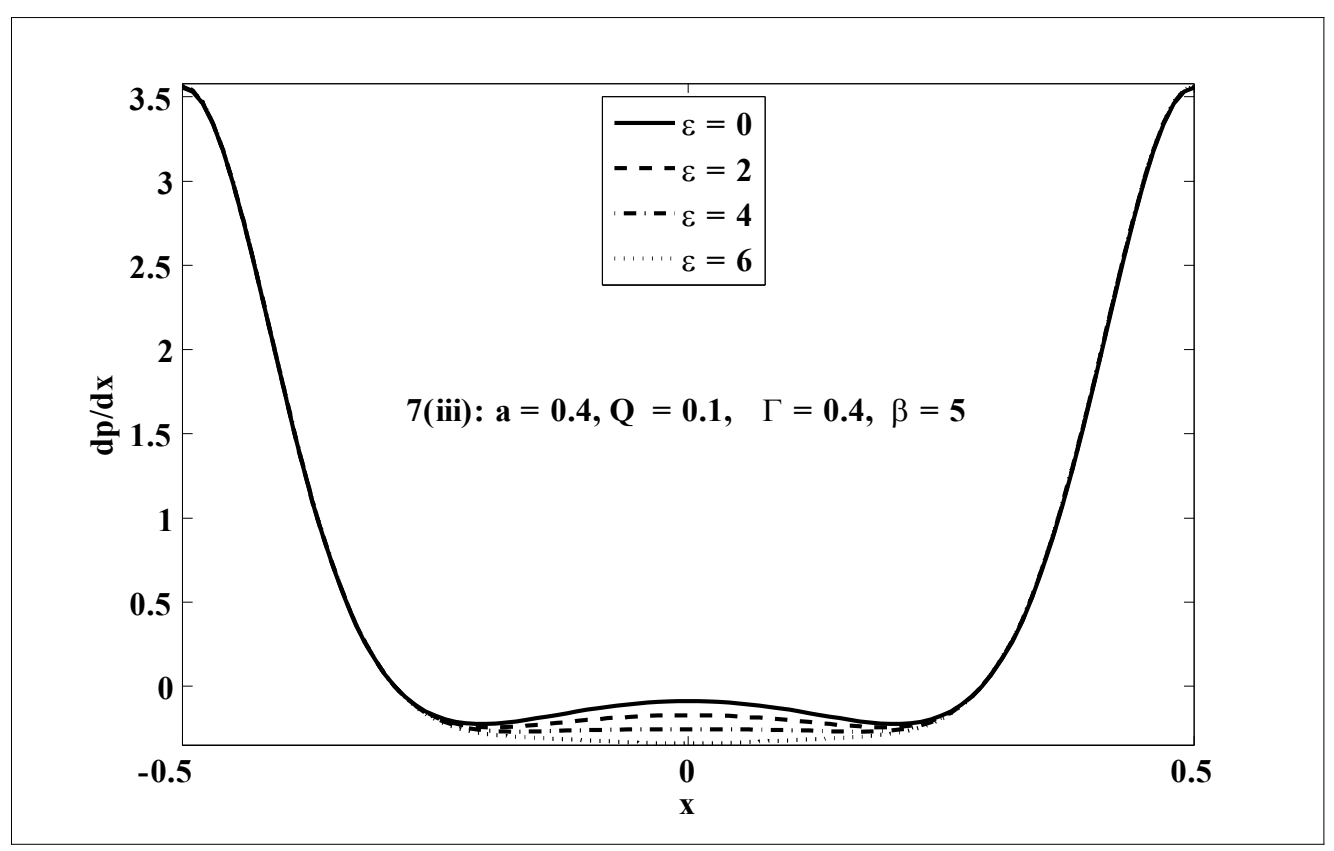

Figure 7. Plots of axial pressure gradient $\Delta P_{\lambda}$ versus $x \in[-0.5,0.5]$.

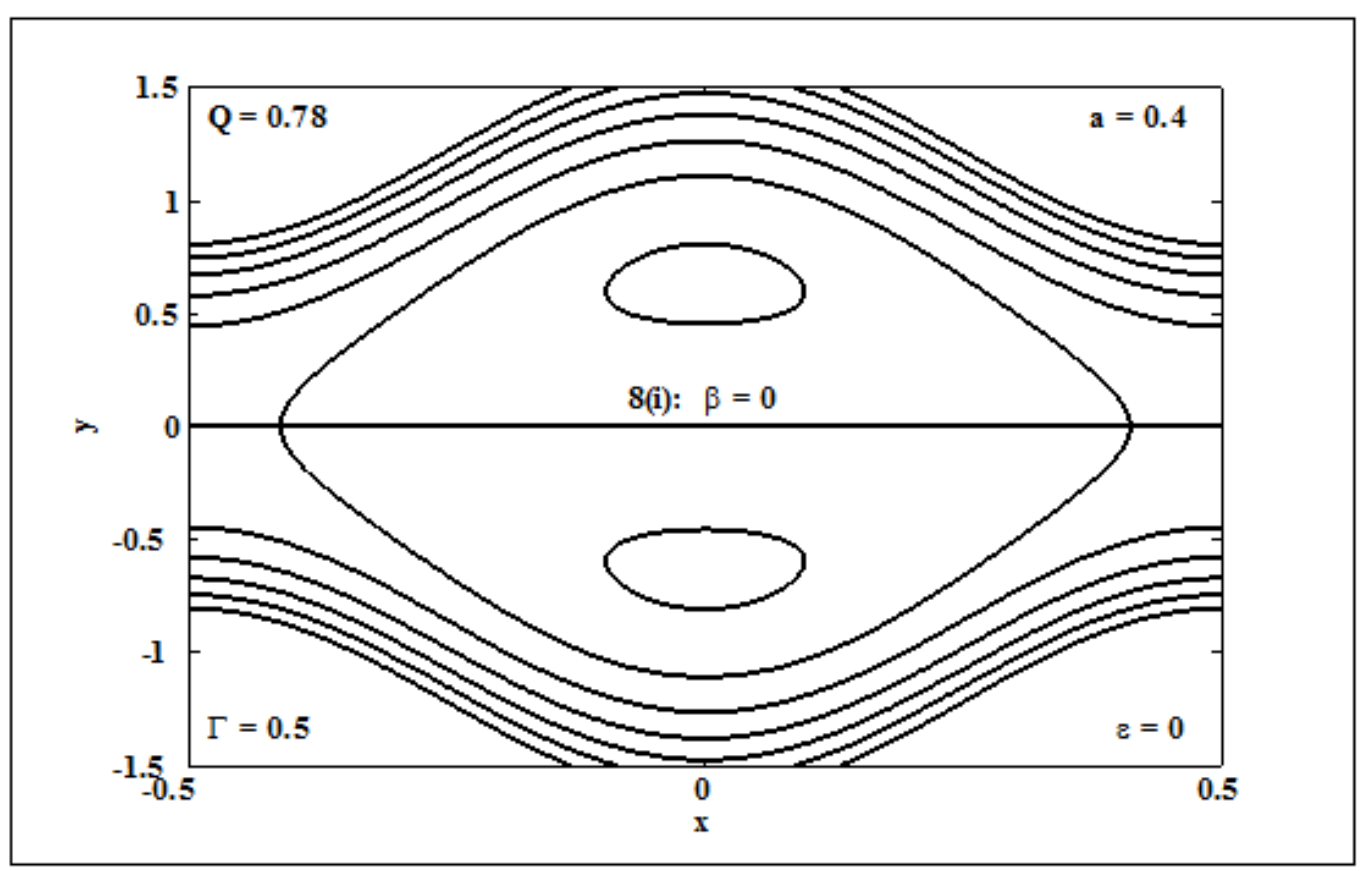



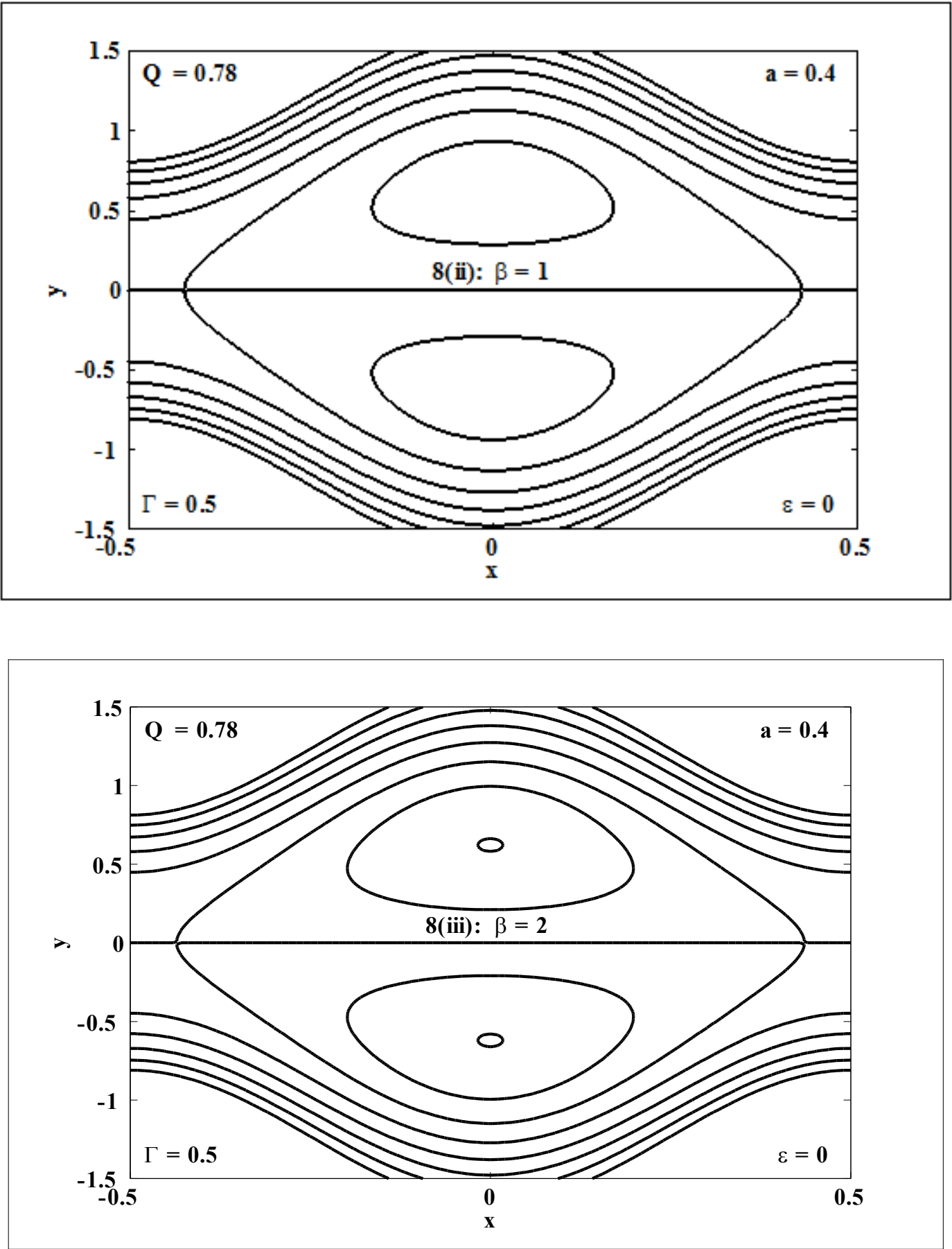

Figure 8. Streamlines for three different values of viscosity parameter $\beta$. 

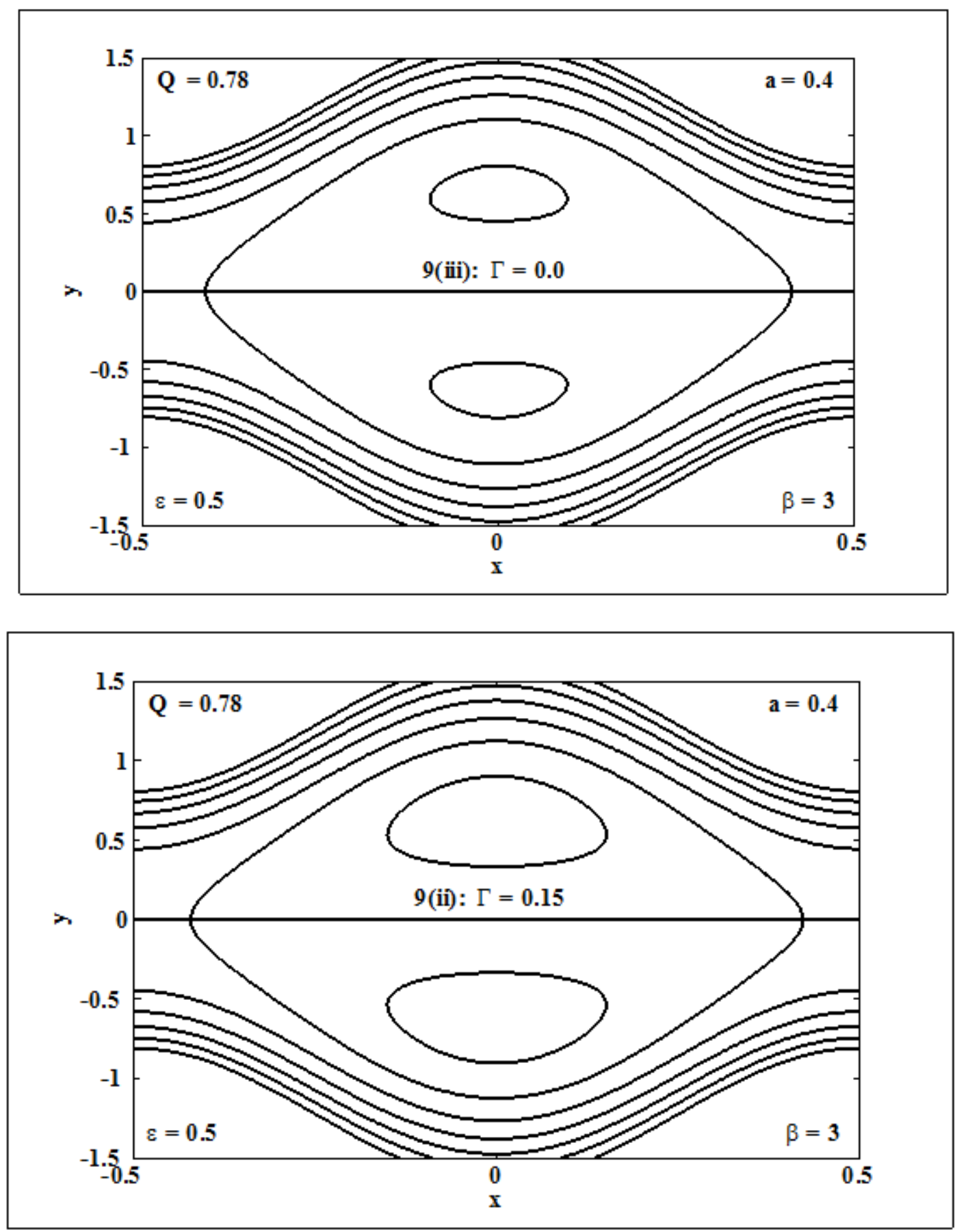


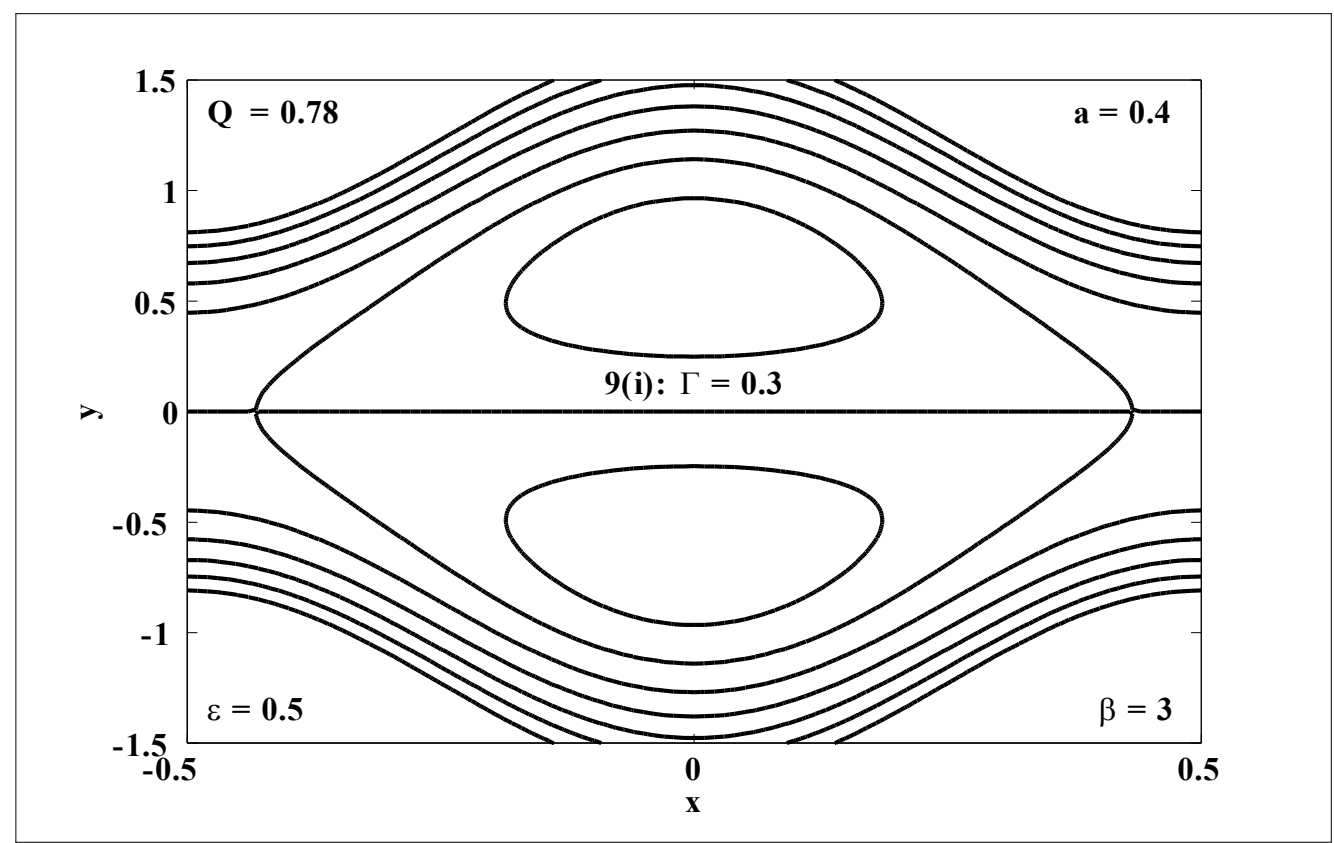

Figure 9. Streamlines for three different values of reaction parameter $\Gamma$.

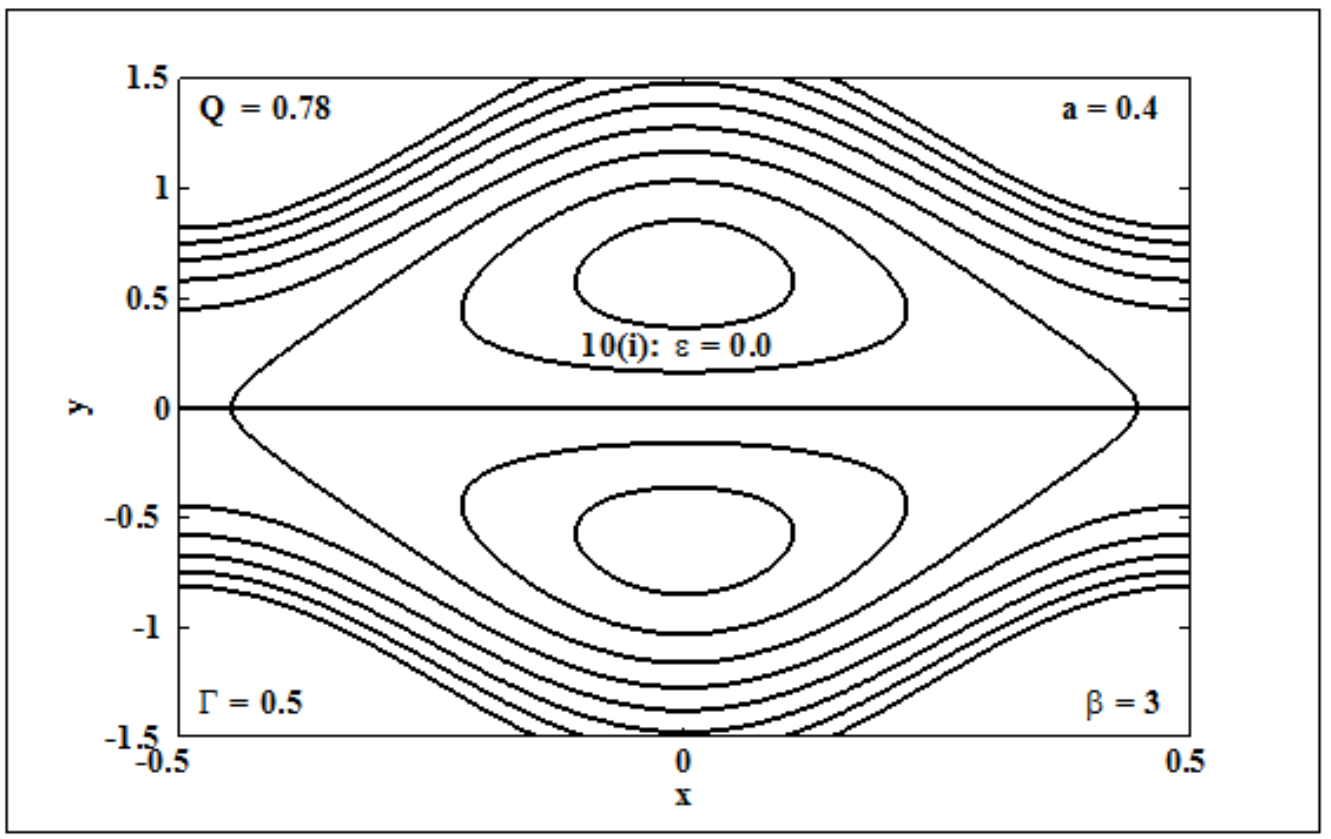



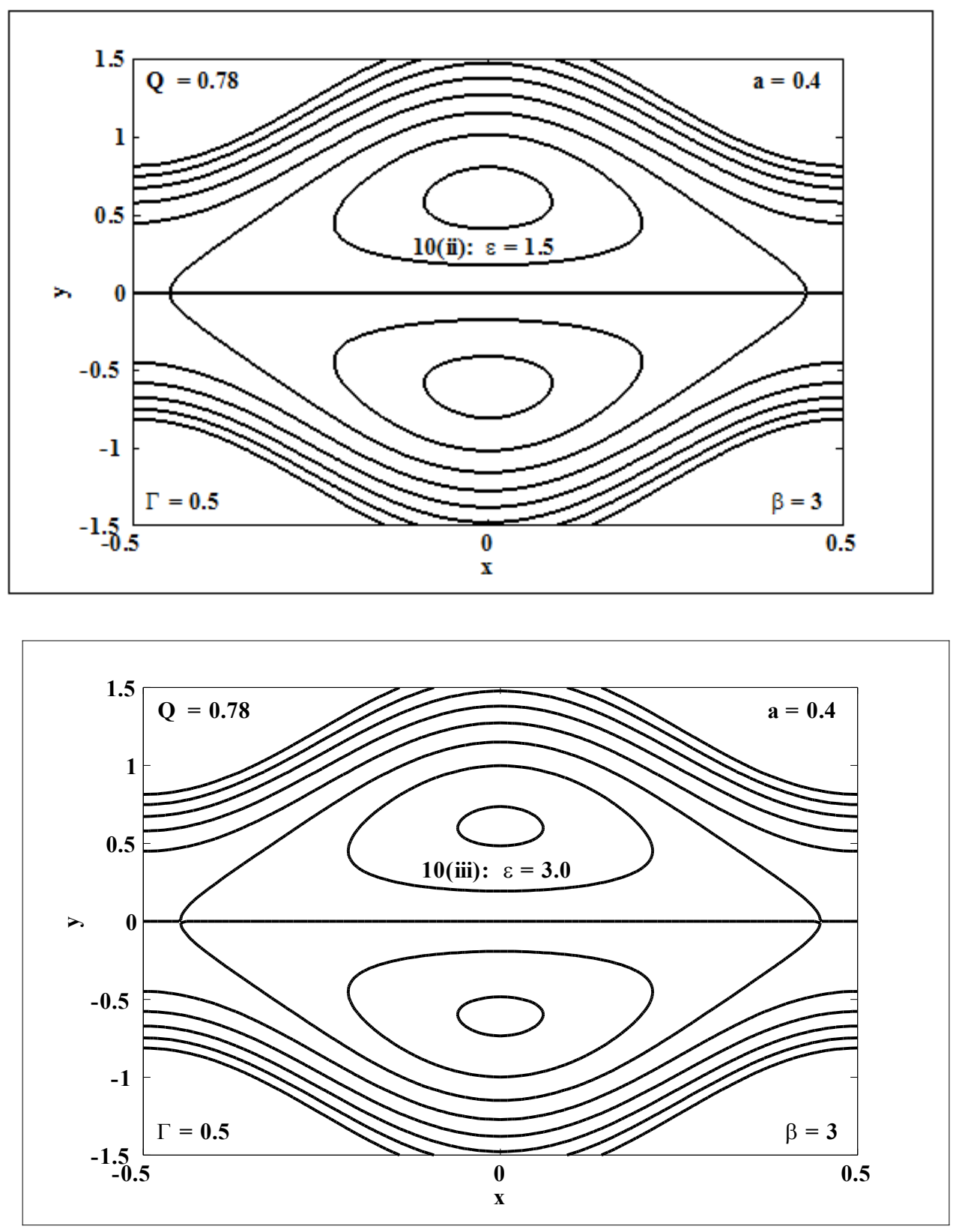

Figure 10. Streamlines for three different values of activation energy parameter $\varepsilon$.

\section{REFERENCES}

1. T. W. Latham, Fluid motion in a peristaltic pump, M.S thesis, Massachusetts Institute of Technology, Cambridge, MA; 1966.

2. J. C. Burns and T. Parkes, Peristaltic motion, Journal of Fluid Mechanics 29, 731743, 1967.

3. A. H. Shapiro, M. Y. Jaffrin and S. L. Weinberg, Peristaltic pumping with long wavelengths at low Reynolds number, Journal of Fluid Mechanics 37, 799-825, 1969.

4. G. Radhakrishnamacharya and Ch. Srinivasulu, Influence of wall properties on 
peristaltic transport with heat transfer, Comptes Rendus Mécanique 335, 369-373, 2007. 5. S. Srinivas and M. Kothandapani, Peristaltic transport in an asymmetric channel with heat transfer - A note, International Journal of Communications in Heat and Mass Transfer 35, 514-522, 2008.

6. Kh. S. Mekheimer and Y. Abd elmaboud, The influence of heat transfer and magnetic field on peristaltic transport of a Newtonian fluid in a vertical annulus: Application of an endoscope, Phyics Letters A 372, 1657-1665, 2008.

7. N. Ali, M. Sajid, T. Javed and Z. Abbas, Heat transfer analysis of peristaltic flow in a curved channel, International Journal of Heat and Mass Transfer 53, 3319-3325, 2010.

8. K. Vajravelu, S. Sreenadh and P. Lakshminarayana, The influence of heat transfer on peristaltic transport of a Jeffrey fluid in a vertical porous stratum, Communications in Nonlinear Science and Numerical Simulation 16, 3107-3125, 2011.

9. D. Tripathi, A mathematical model for swallowing of food bolus through the oesophagus under the influence of heat transfer, International Journal of Thermal Sciences 51, 91-101, 2012.

10. M. Kothandapani and S. Srinivas, On the influence of wall properties in the MHD peristaltic transport with heat transfer and porous medium, Physics Letters A 372, 45864591, 2008.

11. S. Hina, T. Hayat, S. Asghar and A.A. Hendi, Influence of compliant walls on peristaltic motion with heat/mass transfer and chemical reaction, International Journal of Heat and Mass Transfer 55, 3386-3394, 2012.

12. N. S. Akbar, S. Nadeem, T. Hayat, A. A. Hendi, Effects of heat and mass transfer on the peristaltic flow of hyperbolic tangent fluid in an annulus, International Journal of Heat and Mass Transfer 54, 4360-4369, 2011.

13. S. Hina, T. Hayat and A. Alsaedi, Heat and mass transfer effects on the peristaltic flow of Johnson--Segalman fluid in a curved channel with compliant walls, International Journal of Heat and Mass Transfer 55, 3511-3521, 2012.

14. A. El Hakeem A. El Naby, A. E. M. El Misery and I. I. El Shamy, Effects of an endoscope and fluid with variable viscosity on peristaltic motion, Applied Mathematics and Computation 158, 497-511, 2004.

15. E. F. Elshehawey and Z. M. Gharsseldien, Peristaltic transport of three-layered flow with variable viscosity, Applied Mathematics and Computations 153, 417-432, 2004.

16. A. Ebaid, A new numerical solution for the MHD peristaltic flow of a bio-fluid with variable viscosity in a circular cylindrical tube via Adomian decomposition method, Physics Letters A 372, 5321-5328, 2008.

17. N. Ali, Q. Hussain, T. Hayat and S. Asghar, Slip effects on the peristaltic transport of MHD fluid with variable viscosity, Physics Letters A 372, 1477-1489, 2008.

18. T. Hayat and N. Ali, Effect of variable viscosity on the peristaltic transport of a Newtonian fluid in an asymmetric channel, Applied Mathematical Modelling 32, 761774, 2008.

19. S. Nadeem, T. Hayat, N. S. Akbar and M. Y. Malik, On the influence of heat transfer in peristalsis with variable viscosity, International Journal of Heat and Mass Transfer 52, 4722-4730, 2009.

20. S. Nadeem and N.S. Akbar, Influence of temperature dependent viscosity on peristaltic transport of a Newtonian fluid: Application of an endoscope, Applied Mathematics and Computation 216, 3606-3619, 2010. 